\title{
Clinical diagnostic value of combined detection of IMA, D-D and MCP-1 in acute myocardial infarction
}

\author{
MEI DING ${ }^{1 *},{\mathrm{MIN} \mathrm{LI}^{2 *} \text { and HAILING YANG }}^{3}$ \\ Departments of ${ }^{1}$ Cardiology, ${ }^{2}$ Clinical Laboratory, and ${ }^{3}$ Emergency, \\ China-Japan Union Hospital of Jilin University, Changchun, Jilin 130033, P.R. China
}

Received September 18, 2019; Accepted November 25, 2020

DOI: $10.3892 /$ etm.2021.9888

\begin{abstract}
The aim of the study was to analyze the clinical value of the combined detection of ischemia-modified albumin (IMA), D-dimer (D-D) and monocyte chemoattractant protein-1 (MCP-1) in the diagnosis of acute myocardial infarction (AMI). Altogether 87 patients with AMI from January 2017 to January 2018 were enrolled in the AMI group, and 82 patients without coronary artery disease were included in the control group. The serum levels of IMA, D-D, MCP-1, cardiac troponin (CTnT) and high-sensitivity C-reactive protein (hs-CRP) in the two groups were detected by ELISA. The blood lipids of the two groups and the levels of IMA, D-D, MCP-1 after treatment were detected. The association between IMA, D-D, MCP-1, CTnT, hs-CRP and blood lipid in patients with AMI was analyzed. The values of IMA, D-D, and MCP-1 alone and combined in the diagnosis of AMI were analyzed by ROC curve. The levels of IMA, D-D, MCP-1, CTnT and hs-CRP in the AMI group were significantly higher than those in the control group $(\mathrm{P}<0.05)$. The levels of IMA, D-D and MCP-1 in the patients with poor prognosis were significantly higher than those of the good prognosis group $(\mathrm{P}<0.05)$. The changes of IMA, D-D and MCP-1 levels were positively correlated with the levels of CTT and hs-CRP $(\mathrm{P}<0.05)$. The AUC, specificity and sensitivity of patients with AMI diagnosed with MCP-1 alone were $0.8084,81.61$ and $69.51 \%$, respectively. Those of patients diagnosed by D-D were $0.7302,59.77$ and $81.71 \%$, those of patients diagnosed by IMA alone were $0.7289,58.62$ and $80.49 \%$, and those of patients detected by the combination of MCP-1, D-D and IMA were 0.9047, 58.62 and $93.90 \%$. In conclusion, the levels of IMA, D-D and MCP-1 in AMI patients are higher than those in the control group. The
\end{abstract}

Correspondence to: Dr Hailing Yang, Department of Emergency, China-Japan Union Hospital of Jilin University, 126 Xiantai Street, Changchun, Jilin 130033, P.R. China

E-mail: i463uv@163.com; hlyang@jlu.edu.cn

*Contributed equally

Key words: AMI, IMA, D-D, MCP-1, combined detection, clinical prognosis levels of IMA, D-D and MCP-1 were positively correlated with CTnT and hs-CRP levels in AMI patients. Combined detection of IMA, D-D, and MCP-1 can improve the accuracy.

\section{Introduction}

Coronary artery disease can cause imbalance between coronary artery blood flow and myocardial demand, leading to myocardial injury, resulting in myocardial ischemia and hypoxia or necrosis, which is clinically known as 'coronary heart disease' (CHD). Acute myocardial infarction (AMI) is one of the common manifestations of CHD (1). AMI is a kind of myocardial ischemic necrosis caused by coronary artery occlusion and interruption of blood flow. The clinical manifestations of AMI include persistent post-sternal pain, shock, arrhythmia and even death caused by severe heart failure (2). Early diagnosis and treatment of AMI can significantly reduce the mortality and improve the prognosis of patients (3). According to whether there is ST-segment elevation in the ECG, AMI can be divided into 6 types of infarction: Coronary artery bypass graft-related infarction, coronary stent thrombosis-related infarction, percutaneous coronary intervention-related infarction, infarction leading to sudden death without biomarkers, infarction caused by imbalance between supply and demand in blood flow due to causes other than acute atherosclerotic thrombosis, and infarction caused by coronary atherosclerotic thrombosis (1). Epidemiological surveys show that there are approximately 550,000 new AMI and 200,000 relapsed AMI patients in the United States each year, and the global burden of the disease has begun to shift to low- and middle-income countries (4). Approximately $80 \%$ of deaths from cardiovascular diseases come from low- and middle-income countries (4).

Early detection, diagnosis, and treatment are the keys to effectively preventing and reducing the occurrence and death of AMI. Cardiac troponin (cTn) is the first-choice biomarker for AMI detection (2). It has high value in distinguishing unstable angina pectoris, diseases other than acute coronary syndrome and non-ST-segment elevation AMI. The cTn test is highly sensitive to AMI and can improve the sensitivity of diagnosis, but its specificity is low. Its diagnostic performance is affected by diseases such as myocarditis, kidney damage, respiratory failure, and intracranial hemorrhage. Therefore, multi-index combined diagnosis can improve the diagnosis of AMI. 
Ischemia-modified albumin (ische-mia-modified albumin, IMA) is an ideal ischemia marker. It is the first myocardial ischemia marker approved for sale by the US FDA (5). A previous study has confirmed that when myocardial necrosis indicators are negative, IMA can show extremely high sensitivity. For example, IMA can be detected in the blood within 5-10 min of myocardial ischemia. After it reaches a peak value in 1-2 $\mathrm{h}$, IMA returns to the basic level in 3-6 (5). In addition, plasma D-dimer (D-D) and inflammatory cytokine monocyte chemoattractant protein-1 (MCP-1) were detected. As a specific degradation product of cross-linked fibrin, the increase of D-D level reflects the enhancement of secondary fibrinolytic activity, and it can be used as a specific molecular marker for hypercoagulability and fibrinolytic hyperactivity in vivo (6). It has been shown that plasma D-dimer has diagnostic significance in vascular diseases and can be used as one of the auxiliary diagnoses for the determination of AMI (7). MCP-1 is mainly secreted and released by endothelial cells, vascular smooth muscle cells and macrophages. It can induce the expression of certain inflammatory factors, chemokines, matrix metalloproteinases and tissue factors in plaque cells, converting the originally stable plaques into vulnerable plaques, and rupture and secondary thrombosis lead to the occurrence of AMI (8).

The aim of this study was to investigate the application value of the combined detection of IMA, D-D, and MCP-1 for early diagnosis of AMI. The results showed that, the levels of IMA, D-D and MCP-1 were positively correlated with CTnT and hs-CRP levels in AMI patients. Combined detection of IMA, D-D, and MCP-1 can improve the accuracy.

\section{Materials and methods}

General information. A total of 87 patients with AMI who met the diagnostic criteria of AHA/ACC (9) from January 2017 to January 2018 were enrolled in the AMI group, including 53 males and 34 females, with the age of $64.35 \pm 11.90$ years. Another 82 patients who were hospitalized at the same time and confirmed by coronary angiography without coronary artery disease were enrolled in a control group, including 50 males and 32 females, with the age of 59.04 \pm 12.13 years. The general data of patients in the two groups were compared, and there was no statistical difference, as detailed in Table I.

The study was approved by the Medical Ethics Committee of the China-Japan Union Hospital of Jilin University, and informed consent was signed by all the selected subjects.

Inclusion and exclusion criteria. Inclusion criteria were: i) Patients with AMI, diagnosed by AHA/ACC diagnostic criteria; ii) age $\geq 30$ years. Exclusion criteria were: i) Patients with severe liver and kidney insufficiency, tumors and rheumatic diseases; ii) acute or chronic infections; iii) trauma and sports injury.

\section{Inspection indicators}

Determination of serum MCP-1,D-D and IMA content. Fasting venous blood $(5 \mathrm{ml})$ was taken from the selected subjects, and the blood was left to stand for 0.5-1 $\mathrm{h}$ at room temperature. The blood was centrifuged at the speed of $650 \mathrm{x} \mathrm{g}$ at $4^{\circ} \mathrm{C}$ for
$10 \mathrm{~min}$. After centrifugation, the upper serum was retained, and the serum levels of MCP-1, D-D and IMA were detected by ELISA. MCP-1 kit was purchased from ADL Company, and D-D kit was purchased from Merck Biotechnology Co., Ltd. (product no.: cx20026, and item no.: 59-20026). IMA kit was purchased from Shanghai Fusheng Industrial Co., Ltd. (cat. no. A097761-48T). The operation procedure was performed in accordance with the kit instructions.

Determination of CTnT, hs-CRP and blood lipids in patients. Fasting venous blood $(5 \mathrm{ml})$ was taken from the selected subjects, and the blood was let stand for $0.5-1 \mathrm{~h}$ at room temperature. The blood was centrifuged at the speed of $650 \mathrm{xg}$ at $4^{\circ} \mathrm{C}$ for $10 \mathrm{~min}$. After centrifugation, the upper serum was retained, and the serum levels of CTnT and hs-CRP were detected by ELISA. The human CTnT ELISA kit (FKO1147B) was purchased from Shanghai Kexing Biotechnology Co., Ltd., and the human hs-CRP ELISA kit (no. A09718) was purchased from Shanghai Jining Industrial Co., Ltd.. Surgery was performed in accordance with the kit instructions. Total cholesterol (TC), triglyceride (TG), high-density lipoprotein cholesterol (HDL-D) and low-density lipoprotein cholesterol (LDL-C) were measured in the two groups of patients by AU1000/2700 automatic biochemical analyzer.

Prognosis. Conventional treatment was performed on all patients with AMI. There was no significant difference in the treatment. After interventional therapy, oral aspirin enteric-coated tablets were taken at a dose of $100 \mathrm{mg}$ once a day. Captopril (18 mg) and valsartan $(80 \mathrm{mg})$ were taken once a day (10). The patients with AMI after treatment were followed up for one year. Major adverse cardiovascular events (MACE) in AMI patients after treatment were recorded as the group with poor prognosis, while those without MACE were classified as the group with good prognosis. Then serum samples of the two groups were again extracted to measure the contents of MCP-1, D-D and IMA. MACE mainly includes recurrent angina pectoris, AMI, severe arrhythmia, heart failure, death of CHD.

Correlation analysis. Pearson correlation analysis was used to analyze the correlation between MCP-1, D-D, IMA content and TC, TG, HDL-D, LDL-C in patients' blood lipids, and the correlation between MCP-1, D-D, IMA content and prognosis of IMA.

ROC curve analysis. The ROC curve was used to analyze the diagnostic value of IMA, D-D and MCP-1 alone and combined in AMI.

Statistical analysis. SPSS 18.0 software was used to analyze the data, and GraphPad Prism 6 was used to draw all the figures. $\chi^{2}$ test was used to compare the counting data. Mean \pm standard deviation was used to express the measurement data. Independent t-test was used for comparison between the two groups. Paired t-test was used for analysis of data at different time points. Pearson's correlation analysis was used to analyze the relationship between variables. We made the horizontal and vertical co-ordinates according to the sample size, and used SPSS software to generate the ROC curves. Then, AUC values 
Table I. General information.

\begin{tabular}{|c|c|c|c|c|}
\hline Indicators & AMI group (n=87) & Control group $(n=82)$ & $t / \chi^{2}$ & P-value \\
\hline Age & $64.35 \pm 11.90$ & $59.04 \pm 12.13$ & 2.872 & $>0.05$ \\
\hline Sex & & & 0.191 & $>0.05$ \\
\hline Male & $53(60.92)$ & $50(60.98)$ & & \\
\hline Female & $34(39.08)$ & $32(39.02)$ & & \\
\hline Body mass index & $23.67 \pm 3.75$ & $22.74 \pm 3.40$ & 1.590 & 0.113 \\
\hline \multicolumn{5}{|l|}{ Academic level } \\
\hline >high school & $58(66.67 \%)$ & $55(67.07 \%)$ & 0.003 & 0.955 \\
\hline shigh school & $29(33.33 \%)$ & $27(32.93 \%)$ & & \\
\hline Place of domicile & & & 0.001 & 0.999 \\
\hline Rural & $52(59.77 \%)$ & $49(59.76 \%)$ & & \\
\hline City & $35(40.23 \%)$ & $33(40.24 \%)$ & & \\
\hline Smoking & $34(39.08 \%)$ & $30(36.59 \%)$ & 0.112 & 0.738 \\
\hline Family history of CHD & $45(51.72 \%)$ & $40(48.78 \%)$ & 0.146 & 0.702 \\
\hline
\end{tabular}

AMI, acute myocardial infarction; CHD, coronary heart disease.
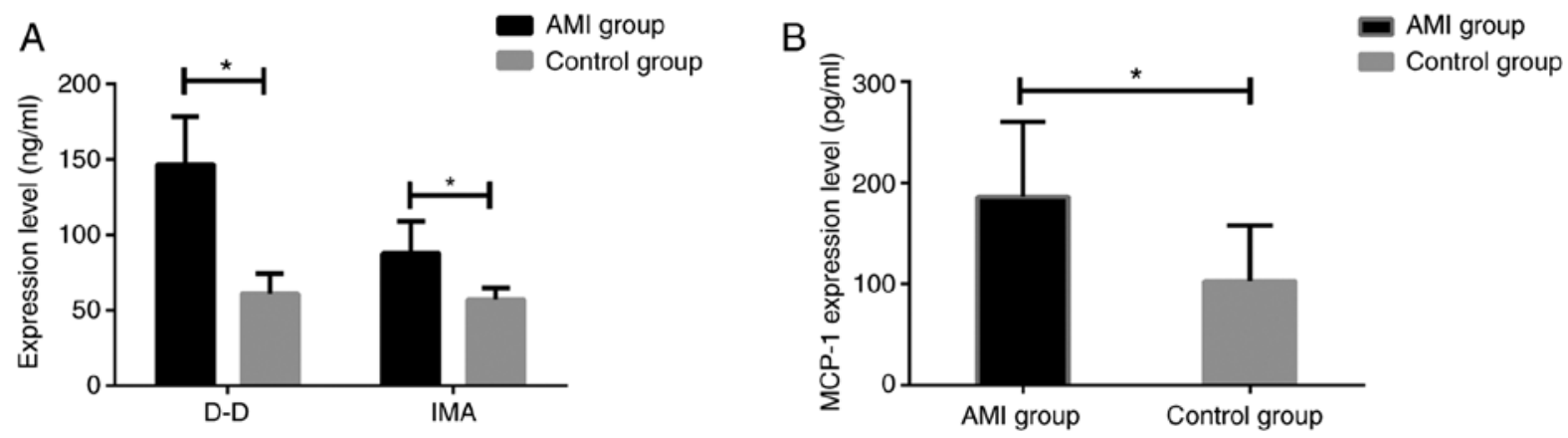

Figure 1. Comparison of serum MCP-1, D-D and IMA levels in two groups. The t-test was used for analysis. (A) ELISA results showed that the serum level of D-D and IMA in the AMI group were significantly higher than those of control group. (B) ELISA results showed that the level of MCP-1 in serum of AMI group was significantly higher than that of control group. * $\mathrm{P}<0.05$. monocyte chemoattractant protein-1; MCP-1, monocyte chemoattractant protein-1; D-D, D-dimer; IMA, ischemia-modified albumin; AMI, acute myocardial infarction.

were determined according to the area under the curve. There was statistical difference when $\mathrm{P}<0.05$.

\section{Results}

Determination of serum MCP-1, D-D and IMA levels in two groups. The expression levels of MCP-1, D-D and IMA in serum of patients in two groups were measured. It was found that the expression of MCP-1, D-D and IMA in patients with AMI was significantly higher than that in the control group (all $\mathrm{P}<0.001$ ) (Fig. 1).

Determination of $C T n T$ and $h s-C R P$ in patients. The levels of CTnT $(55.72 \pm 8.18 \mathrm{ng} / \mathrm{l})$ and hs-CRP $(32.95 \pm 3.62 \mathrm{mg} / \mathrm{l}) \mathrm{in}$ patients with AMI were significantly higher than those in the control group, with CTnT $(0.034 \pm 0.01 \mathrm{ng} / \mathrm{l})$ and hs-CRP $(1.02 \pm 0.35 \mathrm{mg} / \mathrm{l})($ all $\mathrm{P}<0.05)$ (Fig. 2).

Determination of blood lipids in two groups of patients. The blood lipids of the two groups were measured. There were significant differences in TC and LDL-C between AMI group and control group (both $\mathrm{P}<0.05$ ), but no significant differences in TG and HDL-D (both $\mathrm{P}>0.05$ ) (Fig. 3).

Prognosis and serum levels of MCP-1, D-D and IMA in patients with AMI after treatment. After treatment of 87 patients with AMI, 21 cases had recurrent angina pectoris, 7 cases had AMI and 14 cases had severe arrhythmia after one year. After treatment, 42 patients with MACE were classified as poor prognosis group, while 45 patients without MACE were classified as good prognosis group. MCP-1, D-D and IMA in patients with poor prognosis were detected within $48 \mathrm{~h}$ of MACE and the data were retained. At the end of the follow-up period, MCP-1, D-D and IMA in patients with good prognosis without MACE were detected, and the data of two groups were compared. The levels of MCP-1 (168.72 \pm 53.31$)$, D-D $(113.04 \pm 37.47)$, IMA $(77.61 \pm 20.15)$ in patients with poor prognosis were significantly higher than those in patients with

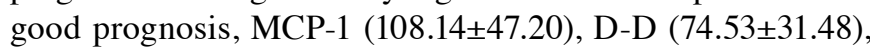
IMA (62.21 \pm 21.47$)($ all $\mathrm{P}<0.05)$ (Fig. 4). 
Table II. ROC curve analysis.

\begin{tabular}{|c|c|c|c|c|c|c|}
\hline Indicators & AUC & Sensitivity (\%) & Specificity (\%) & Standard error & $95 \% \mathrm{CI}$ & $\mathrm{P}$-value \\
\hline MCP-1 & 0.8084 & 81.61 & 69.51 & 0.03373 & $0.7423-0.8745$ & $<0.001$ \\
\hline D-D & 0.7302 & 59.77 & 81.71 & 0.03832 & $0.6550-0.8053$ & $<0.001$ \\
\hline IMA & 0.7289 & 58.62 & 80.49 & 0.03830 & $0.6538-0.8040$ & $<0.001$ \\
\hline MCP-1, D-D, IMA & 0.9047 & 58.62 & 93.90 & 0.02249 & $0.8606-0.9488$ & $<0.001$ \\
\hline
\end{tabular}

MCP-1, monocyte chemoattractant protein-1; D-D, D-dimer; IMA, ischemia-modified albumin; AMI, acute myocardial infarction.
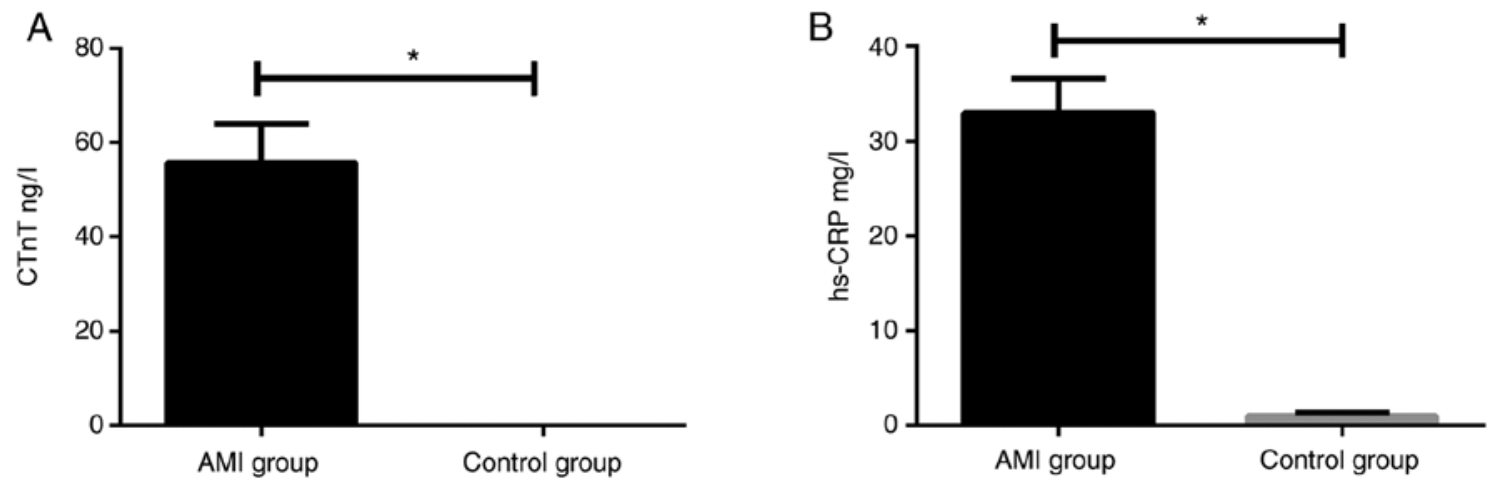

Figure 2. Comparison of CTnT and hs-CRP levels in the two groups of patients. The t-test was used for analysis. (A) ELISA results showed that the serum levels of CTnT in the AMI group were significantly higher than those in the control group. (B) ELISA results showed that the serum levels of hs-CRP in the AMI group were significantly higher than that in the control group. ${ }^{~} \mathrm{P}<0.05$. cTnT, cardiac troponin; hs-CRP, high-sensitivity $\mathrm{C}$-reactive protein; AMI, acute myocardial infarction.

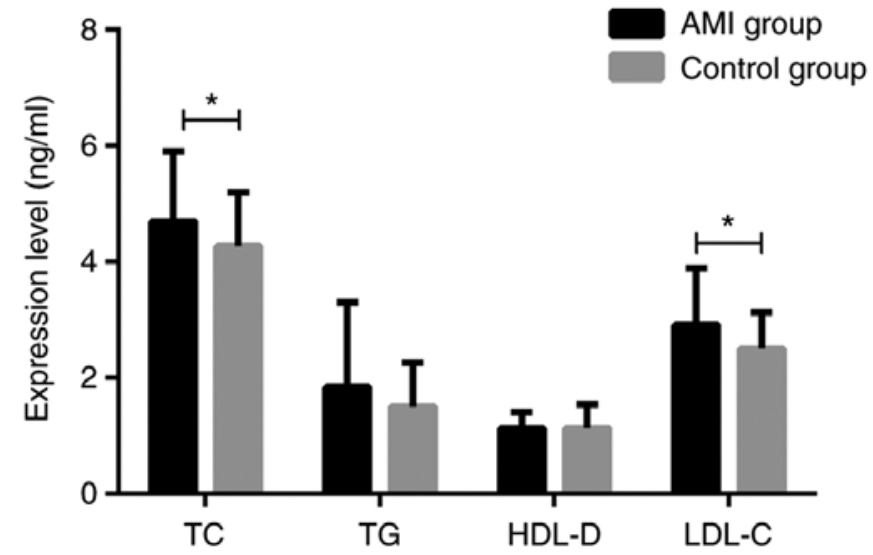

Figure 3. Comparison of the expression levels of blood lipids in the two groups of patients. The t-test was used for analysis. There was a statistically significant difference in TC and LDL-C (both $\mathrm{P}<0.05$ ), while there was no significant difference between TG and HDL-D (both $\mathrm{P}>0.05$ ) between the AMI group and the control group. ${ }^{*} \mathrm{P}<0.05$. TC, total cholesterol; TG, triglyceride; LDL-C, low-density lipoprotein cholesterol; HDL-D, high-density lipoprotein cholesterol; AMI, acute myocardial infarction.

Correlation analysis of serum MCP-1, D-D and IMA levels. Pearson's correlation analysis was used to analyze the correlation between MCP-1, D-D, IMA and TC, TG, HDL-D, LDL-C in patient's blood lipids. The correlation between MCP-1, D-D, IMA content and prognosis of IMA was analyzed. The index levels measured by the AMI group were used as MCP-1, D-D, and IMA content.
There was no correlation between MCP-1, D-D, IMA levels and TC, TG, HDL-D, LDL-C levels in serum lipids in patients with AMI (all P>0.05) (Fig. 5). The serum levels of MCP-1, D-D and IMA were positively correlated with the levels of CTnT and hs-CRP in patients with AMI. Fig. 5A: $r=0.6712$, $\mathrm{P}=<0.001$; Fig. 5B: $\mathrm{r}=0.4492, \mathrm{P}=<0.001$; Fig. 5C: $\mathrm{r}=0.4547$, $\mathrm{P}=<0.001$; Fig. 5D: $\mathrm{r}=0.5538, \mathrm{P}=<0.001$; Fig. 5E: $\mathrm{r}=0.5607$, $\mathrm{P}=<0.001$. Fig. 5F: $\mathrm{r}=0.5070, \mathrm{P}=<0.001$ (all $\mathrm{P}<0.05$ ) (Fig. 6).

ROC curve analysis. The AUC was 0.8084 for patients with AMI diagnosed alone with MCP-1, the specificity was $81.61 \%$, and the sensitivity was $69.51 \%$. The AUC was 0.7302 for patients with AMI diagnosed alone with D-D, the specificity was $59.77 \%$, and the sensitivity was $81.71 \%$. The AUC was 0.7289 for AMI patients diagnosed alone with IMA, the specificity was $58.62 \%$, and the sensitivity was $80.49 \%$. The AUC was 0.9047 in combined detection of MCP-1, D-D and IMA in patients with AMI, the specificity was $58.62 \%$, and the sensitivity was $93.90 \%$. Other data are shown in Table II, and Figs. 7 and 8 .

\section{Discussion}

Patients with AMI are more likely to have coronary atherosclerotic stenosis. Due to certain inducements, atherosclerotic plaque ruptures. Platelets in the blood gather on the surface of the ruptured plaque, forming blood clots, and eventually obstruct the coronary artery lumen. In addition, AMI can also be induced by a sharp increase in myocardial oxygen 

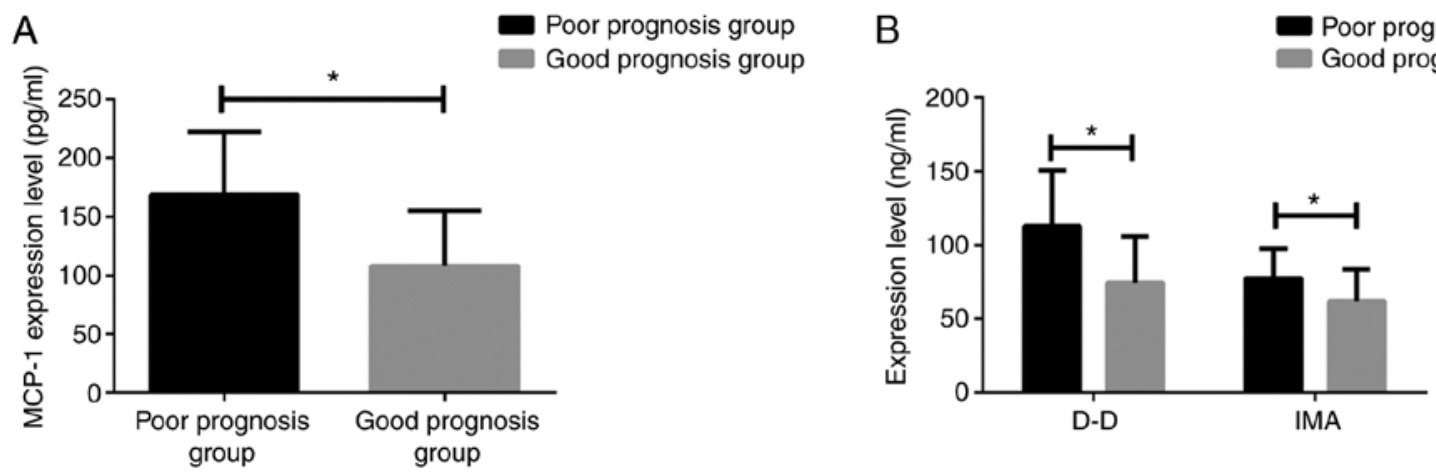

Figure 4. Comparison of serum MCP-1, D-D and IMA levels in patients with AMI prognosis group. The t-test was used for analysis. (A) ELISA results showed that MCP-1 levels in patients with poor prognosis were significantly higher than those in good prognosis group. (B) ELISA results showed that the levels of D-D and IMA in the patients with poor prognosis were significantly higher than those in the good prognosis group. " $\mathrm{P}<0.05$. MCP-1, monocyte chemoattractant protein-1; D-D, D-dimer; IMA, ischemia-modified albumin; AMI, acute myocardial infarction.

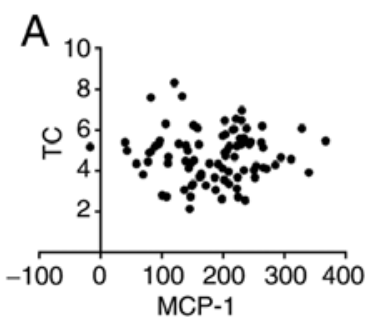

$\mathrm{E}$

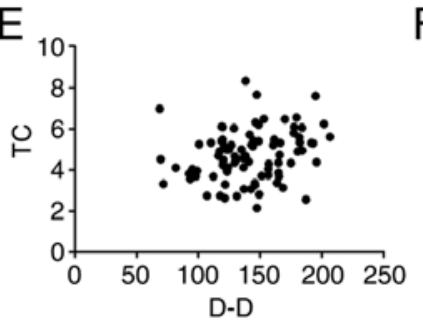

I

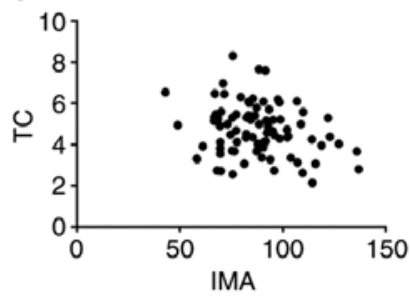

B

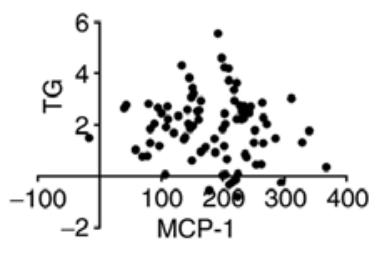

F

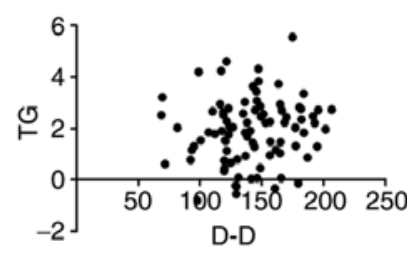

J

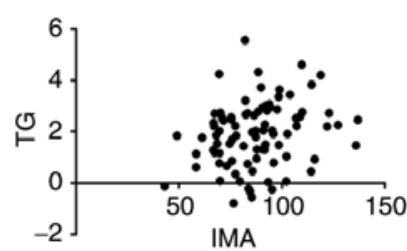

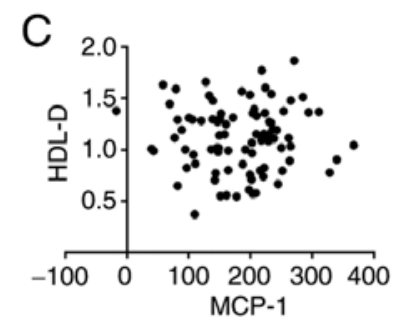

G

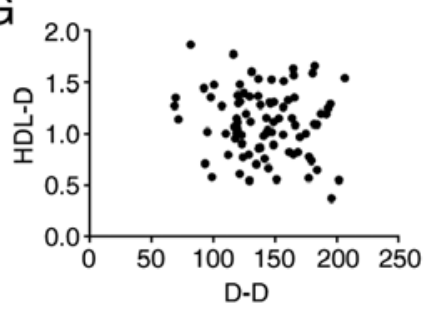

K

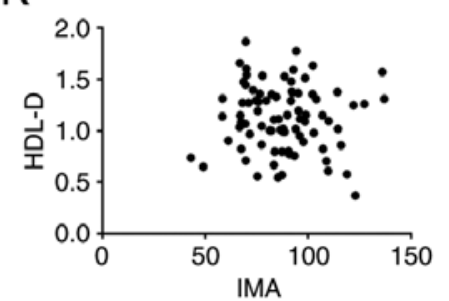

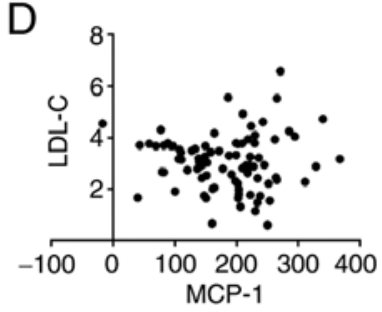

$\mathrm{H}$

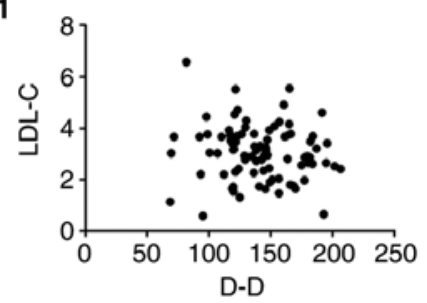

L

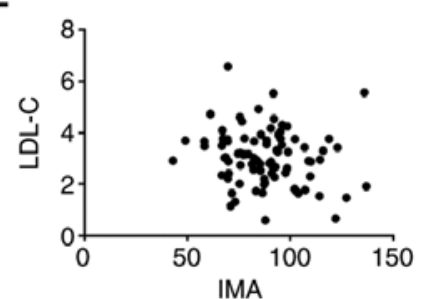

Figure 5. Correlation analysis of serum levels of MCP-1, D-D, IMA and TC, TG, HDL-D, and LDL-C levels in blood lipids. Pearson's correlation analysis showed that serum levels of MCP-1 (A-D), D-D (E-H), and IMA (I-L) were not correlated with TC, TG, HDL-D, and LDL-C levels in blood lipids (all P>0.05). MCP-1, monocyte chemoattractant protein-1; D-D, D-dimer; IMA, ischemia-modified albumin; TC, total cholesterol; TG, triglyceride; HDL-D, high-density lipoprotein cholesterol; LDL-C, low-density lipoprotein cholesterol.

consumption or coronary artery spasm. Sudden cold stimulation may induce myocardial infarction. Approximately half of the patients often missed diagnosis and delayed the best time of treatment because there was no obvious changes in early ECG. Early detection of AMI can effectively improve the treatment and prognosis of patients $(11,12)$.

MCP-1 (13) is a cellular inflammatory factor secreted by monocytes, with multiple thiol and hydroxyl groups. It can promote the activation of downstream monocytes or macrophages and accelerate the formation of local microvessels on the basis of binding glycoprotein ligands. In a related study by Niu J (14), it was concluded that many diseases including cardiovascular diseases are considered to be inflammatory diseases, and MCP-1 plays a key role in the development of cardiovascular diseases. MCP-1 also works in cardiac repair and participates in ischemic angiogenesis. The results of this experiment showed that MCP-1 is highly expressed in AMI.

D-D $(15,16)$ is a molecular marker of secondary fibrinolysis and hypercoagulability. D-D increases due to the formation of activated thrombosis and the presence of fibrinolysis activity in the early stage of myocardial infarction. This study showed that the level of D-D in serum of patients with AMI was significantly higher than that of the control group, indicating that there is a potential link between D-D and AMI. However, D-D 

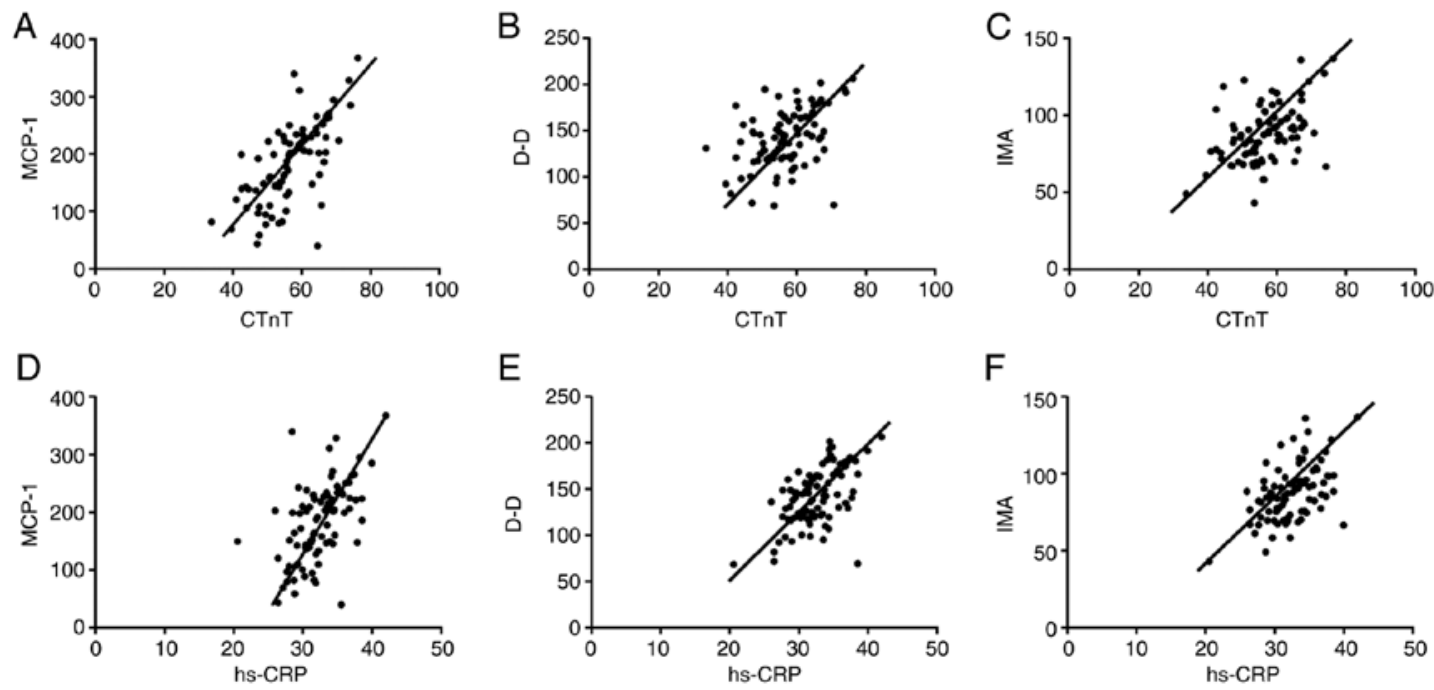

Figure 6. Correlation analysis of MCP-1, D-D, IMA levels and CTnT, hs-CRP levels. (A) MCP-1 is positively correlated with CTnT, $\mathrm{r}=0.6712$. (B) D-D is positively correlated with $\mathrm{CTnT}, \mathrm{r}=0.4492$. (C) IMA is positively correlated with $\mathrm{CTnT}, \mathrm{r}=0.4547$. (D) MCP-1 is positively correlated with hs-CRP, $\mathrm{r}=0.5538$. (E) D-D is positively correlated with hs-CRP, $r=0.5607$. (F) IMA is positively correlated with hs-CRP, $r=0.5070$ (all $\mathrm{P}<0.05$ ). MCP-1, monocyte chemoattractant protein-1; D-D, D-dimer; IMA, ischemia-modified albumin; cTnT, cardiac troponin; hs-CRP, high-sensitivity C-reactive protein.
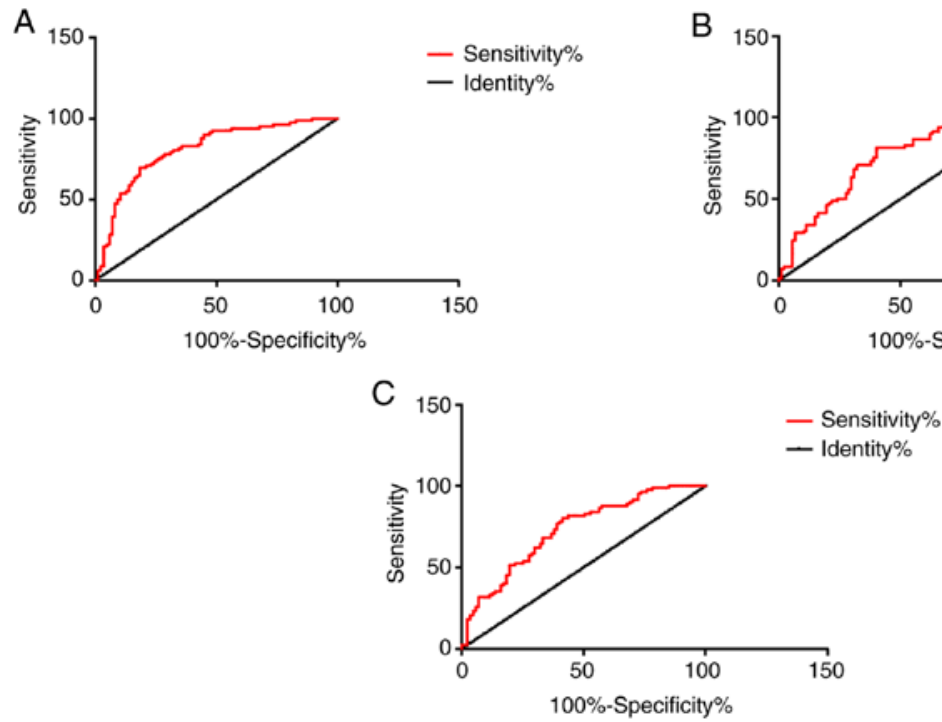

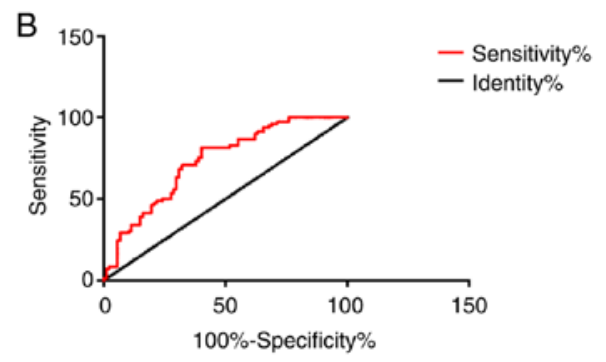

Figure 7. ROC curves of MCP-1, D-D and IMA for detecting AMI independently. (A) The AUC of patients with AMI diagnosed alone with MCP-1 was 0.8084 the specificity was $81.61 \%$, and the sensitivity was $69.51 \%$. (B) The AUC of patients with AMI diagnosed alone with D-D was 0.7302 , the specificity was $59.77 \%$, and the sensitivity was $81.71 \%$. (C) The AUC of AMI patients diagnosed alone with IMA was 0.7289 , the specificity was $58.62 \%$, and the sensitivity was $80.49 \%$. MCP-1, monocyte chemoattractant protein-1; D-D, D-dimer; IMA, ischemia-modified albumin; AMI, acute myocardial infarction.

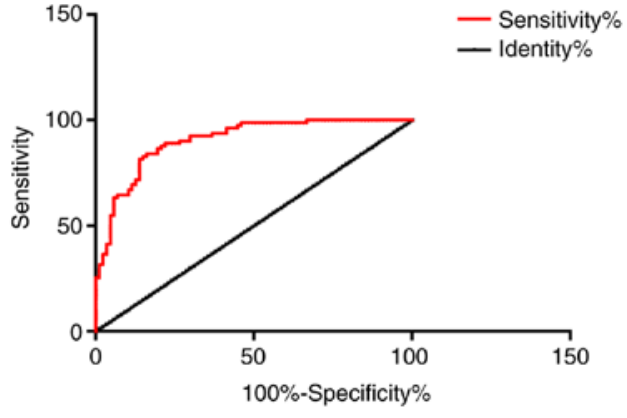

Figure 8. The AUC of combined detection of MCP-1, D-D and IMA in patients with AMI was 0.9047 , the specificity was $58.62 \%$, and the sensitivity was $93.90 \%$. MCP-1, monocyte chemoattractant protein-1; IMA, ischemia-modified albumin; AMI, acute myocardial infarction. was also increased in other diseases (17). ROC curve analysis showed that D-D was not specific for AMI and it could not be used as an independent predictor.

IMA (18) was discovered in the 1990s as a serum albumin damaged by hydroxyl radicals during myocardial ischemia. Serum albumin is known as IMA when the 2-4 amino acid residues at the $\mathrm{N}$-terminus of serum albumin are deleted by $\mathrm{N}$-acetylation and then bound to metallic cobalt. Sokhanvar S (19) found that IMA can be used as a diagnostic factor for acute coronary syndrome (ACS). Although its sensitivity and specificity were not higher compared with other diagnostic tests, it still has diagnostic value for coronary disease. The results of this experiment show that the level of IMA in serum of patients with AMI is significantly higher 
than that of control group. These results suggest that IMA can be used as an independent criterion for increased risk of AMI, which is consistent with the results of the study by Zhong et al (20).

At the same time, this paper also selected two common indicators of AMI: Cardiac troponin (cTnT) and high-sensitivity C-reactive protein (hs-CRP). CTnT is considered to be the most ideal early diagnostic index for AMI at present, and it is also an important index for patients' disease evaluation, prognosis judgment and curative effect observation (21). hs-CRP is one of the risk indicators for cardiovascular and cerebrovascular diseases (22). The results of this study showed that the levels of cTnT and hs-CRP in the patients with AMI were significantly higher than those in the control group, which was consistent with the results of Keller and Han (23,24). It was found that MCP-1, D-D and IMA were highly expressed in patients with AMI. It was speculated that MCP-1, D-D and IMA were potentially associated with cTnT and hs-CRP. Pearson correlation analysis showed that serum MCP-1, D-D, IMA levels were positively correlated with CTnT and hs-CRP levels in patients with AMI. Patients were followed up for one year after treatment. Patients received routine treatment, so there is no difference in the effect of treatment on prognosis. According to the occurrence of MACE, patients were divided into group with the poor prognosis and group with good prognosis, and the levels of MCP-1, D-D and IMA in the two groups were detected. The results showed that the levels of MCP-1, D-D and IMA in patients with poor prognosis were significantly higher than those in patients with good prognosis, suggesting that detection of MCP-1, D-D and IMA expression levels can predict the prognosis of patients with AMI.

The ROC curves of patients with MCP-1, D-D and IMA were analyzed. The AUC of single detection of AMI was 0.8084, 0.7302 and 0.7289, respectively. However, the AUC of three combined detection was 0.9047 , and the specificity and sensitivity were significantly improved, suggesting that combined detection can improve the accuracy of diagnosis of AMI, so misdiagnosis and missed diagnosis could be reduced. However, this study also has certain limitations, such as the small sample size. Moreover, the combined detection of MCP-1, D-D and IMA in this study can only be used as a complementary diagnosis, but not as a substitute for coronary angiography. This experiment failed to make a deeper exploration of the pathogenesis of AMI, and hope that in-depth research will be conducted in the future. We did not collect multicenter data in this study, but multicenter data will be collected in the future.

In conclusion, combined detection of MCP-1, D-D and IMA can effectively improve the diagnostic rate of AMI, detect the levels of IMA, D-D and MCP-1 to predict the prognosis of patients, and suggest routine joint detection in clinical departments.

\section{Acknowledgements}

Not applicable.

\section{Funding}

No funding was received.

\section{Availability of data and materials}

The datasets used and/or analyzed during the present study are available from the corresponding author on reasonable request.

\section{Authors' contributions}

MD and ML conceived and designed the study, and drafted the manuscript. MD, ML and HY collected, analyzed and interpreted the experimental data. HY revised the manuscript for important intellectual content. All authors read and approved the final manuscript.

\section{Ethics approval and consent to participate}

The study was approved by the Ethics Committee of China-Japan Union Hospital of Jilin University. Signed written informed consents were obtained from the patients and/or guardians.

\section{Patient consent for publication}

Not applicable.

\section{Competing interests}

The authors declare that they have no competing interests.

\section{References}

1. Boersma E, Mercado N, Poldermans D, Gardien M, Vos J and Simoons ML: Acute myocardial infarction. Lancet 361: 847-858, 2003.

2. Rumana N, Kita Y, Turin TC, Murakami Y, Sugihara H, Morita Y, Tomioka N, Okayama A, Nakamura Y, Abbott RD and Ueshima $\mathrm{H}$ : Trend of increase in the incidence of acute myocardial infarction in a Japanese population: Takashima AMI registry, 1990-2001. Am J Epidemiol 167: 1358-1364, 2008.

3. Ferrières J, Cambou JP, Ruidavets JB and Pous J: Trends in acute myocardial infarction prognosis and treatment in southwestern France between 1985 and 1990 (the MONICA Project-Toulouse). Am J Cardiol 75: 1202-1205, 1995

4. Anderson JL and Morrow DA: Acute myocardial infarction. N Engl J Med 376: 2053-2064, 2017.

5. Açıkgöz Ș, Edebali N, Barut F, Can M, Tekin İÖ, Büyükuysal Ç and Açıkgöz B: Ischemia modified albumin increase indicating cardiac damage after experimental subarachnoid hemorrhage. BMC Neurosci 15: 33, 2014.

6. Tripodi A. D-dimer testing in laboratory practice. Clin Chem 57: 1256-1262, 2011.

7. Andreescu AC, Cushman M and Rosendaal FR: D-dimer as a risk factor for deep vein thrombosis: The Leiden thrombophilia study. Thromb Haemost 87: 47-51, 2002.

8. Lloyd CM, Minto AW, Dorf ME, Proudfoot A, Wells TN, Salant DJ and Gutierrez-Ramos JC: RANTES and monocyte chemoattractant protein-1 (MCP-1) play an important role in the inflammatory phase of crescentic nephritis, but only MCP-1 is involved in crescent formation and interstitial fibrosis. J Exp Med 185: 1371-1380, 1997.

9. Ryan TJ, Antman EM, Brooks NH, Califf RM, Hillis LD, Hiratzka LF, Rapaport E, Riegel B, Russell RO, Smith EE III, et al: 1999 update: ACC/AHA guidelines for the management of patients with acute myocardial infarction. A report of the American college of cardiology/American heart association task force on practice guidelines (committee on management of acute myocardial infarction). J Am Coll Cardiol 34: 890-911, 1999.

10. Gong X, Zhou R and Li Q: Effects of captopril and valsartan on ventricular remodeling and inflammatory cytokines after interventional therapy for AMI. Exp Ther Med 16: 3579-3583, 2018. 
11. Rubinfeld GD, Smilowitz NR, Berger JS and Newman JD Association of thrombocytopenia, revascularization, and in-hospital outcomes in patients with acute myocardial infarction. Am J Med 132: 942-948.e5, 2019.

12. Rasmussen MB, Stengaard C, Sørensen JT, Riddervold IS Hansen TM, Giebner M, Rasmussen CH, Bøtker HE and Terkelsen CJ: Predictive value of routine point-of-care cardiac troponin $\mathrm{T}$ measurement for prehospital diagnosis and risk-stratification in patients with suspected acute myocardial infarction. Eur Heart J Acute Cardiovasc Care 8: 299-308, 2019.

13. Gruber HE, Hoelscher GL, Ingram JA, Bethea S, Cox M and Hanley EN Jr: Proinflammatory cytokines modulate the chemokine CCL2 (MCP-1) in human annulus cells in vitro: CCL2 expression and production. Exp Mol Pathol 98: 102-105, 2015.

14. Niu J and Kolattukudy PE: Role of MCP-1 in cardiovascular disease: Molecular mechanisms and clinical implications. Clin Sci (Lond) 117: 95-109, 2009.

15. Buitrago L, Zafar H, Zhang Y, Li J, Walz T and Coller BS: Dominant role of $\alpha \operatorname{IIb} \beta 3$ in platelet interactions with cross-linked fibrin fragment D-dimer. Blood Adv 4: 2939-2949, 2020.

16. Simes J, Robledo KP, White HD, Espinoza D, Stewart RA Sullivan DR, Zeller T, Hague W, Nestel PJ, Glasziou PP, et al: D-dimer predicts long-term cause-specific mortality, cardiovascular events, and cancer in patients with stable coronary heart disease: LIPID Study: LIPID Study. Circulation 138: 712-723, 2018.

17. Shaw E, Massaro J, Levy D, O'Donnell CJ, D'Agostino R and Tofler G: D-dimer and the risk of cardiovascular disease: The Framingham heart study. Heart Lung Circ 20 (Suppl 2): S30, 2011.
18. Kim KS, Shin SD, Song KJ, Suh GJ and Shin S: Prognosis of patients with out-of-hospital cardiac arrest and early biochemical markers: Ischemia modified albumin, procalictonin, and S-100 protein. J Korean Soc Emerg Med 17: 281-290, 2006.

19. Sokhanvar S, Mellati AO, Mousavinasab SN, Taran L, Vahdani B and Golmmohamadi Z: Ischemia-modified albumin (IMA) in differential diagnosis of transient myocardial ischemia from non ischemic chest pain. Bratisl Lek Listy 113: 612-615, 2012.

20. Zhong Y, Wang $\mathrm{N}$ and $\mathrm{Xu} \mathrm{H}$ : The value of ischaemia modified albumin in the diagnosis on coronary atherosclerotic heart disease. Heart 97 (Suppl 3): A134, 2011.

21. Zhang CL, Jiang YM, Gao X and Wang X: Clinical value of h-FABP, hs-CRP, cTnT examination to diagnose acute myocardial infarction. J Dalian Med Univ 30: 170-172, 2008.

22. Devaki RN, Suma MN, Gowdappa HB, et al: HS-CRP levels in myocardial infarction patients in relation to cardiac markers, 2011.

23. Keller T, Zeller T, Peetz D, Tzikas S, Roth A, Czyz E, Bickel C, Baldus S, Warnholtz A, Fröhlich M, et al: Sensitive troponin I assay in early diagnosis of acute myocardial infarction. N Engl J Med 361: 868-877, 2009.

24. Chan D and Ng LL: Biomarkers in acute myocardial infarction. BMC Med 8: 34, 2010.

This work is licensed under a Creative Commons

Attribution-NonCommercial-NoDerivatives 4.0 International (CC BY-NC-ND 4.0) License. 